\title{
Important Properties of Grapes and Wine from the Dobrogea Area for Therapeutic Use
}

\author{
Rodica Sirbu \\ "Ovidius" University of Constanta, Faculty of \\ Pharmacy, Constanta, Romania \\ Ticuta Negreanu-Pirjol \\ "Ovidius" University of Constanta, Faculty of \\ Pharmacy, Constanta, Romania \\ Bogdan Stefan Negreanu-Pirjol \\ "Ovidius" University of Constanta, Faculty of \\ Pharmacy, Constanta, Romania \\ Emin Cadar \\ "Ovidius" University of Constanta, Faculty of \\ Pharmacy, Constanta, Romania
}

\begin{abstract}
Starting from Herodotus who states that: No cure can cure like wine and can not inspire like it. From antiquity until now grapes and wine represent a viable alternative in multiple therapies if we know how and when to consume these products. This paper is a study that comes to present concretely the beneficial impact of the products that the vine offers us, the plant cultivated in the location of our Dobrogean area. Grapes contain reducing sugars, malic acid, succinic acid, flavonic derivatives, lecithin, choline, leucine, wax and minerals. Wine also has special properties in cellular and mental balance, being a good anti-stress remedy. Medicinal wines are elixirs with beneficial effects on health only if they are prepared correctly. In this paper important properies of wine and grapes are analised and application of wines and their specific effects in different therapies was systematized. The paper aims to open interest in this topic for scientifically controlled consumption and for the use of these natural products in beneficial alternative therapies.
\end{abstract}

Keywords: wine, grapes, sugar content, polyphenols content, total acidity, anthocyanins content 


\section{Introduction}

In ancient Greece, Hippocrates, the father of medicine, recommended the wine of those with pneumonia, physical weakness, in the treatment of open wounds, but also as an antidote if someone was bitten by a venomous snake. Grape belts are suitable for both healthy and especially sick people. Grapes contain reducing sugars, malic acid, succinic acid, flavonic derivatives, lecithin, choline, leucine, wax and minerals. The Dacians used wine in the preparation of eye ointments. Today, the virtues of wine are well known: invigorating, euphoric, vasodilator and tranquilizer. Its therapeutic properties do not stop here, the wine also having antiviral action, especially against polio virus, liver virus and enteroviruses [1]. About wine we know that it helps lower cholesterol and, consumed in moderation, even helps increase good cholesterol. The polyphenols contained in wine protect the walls of blood vessels. The condition is that they should be consumed in moderate quantities $(1-2$ or at most 3 glasses a day, at the table), for long periods of time and, obligatorily, from a natural wine [1]. A study published in the British Medical Journal [2] says that those who consume up to seven glasses of wine a week have a $30 \%$ lower risk of having a stroke. The study was conducted on 599,912 consumer respondents taken from 19 high-income UK countries that do not have a history of cardiovascular disease. The wine has special properties in the cellular and mental balance, being a good anti-stress remedy. Wine is also a medicine for women, but consumed more by men, experts say.

\section{Material and Methods}

The most representative varieties are from the Murfatlar Viticulture and Vinification Research and Development Station. The qualities of grapes and wines were analyzed on these varieties. Among the white varieties, Chardonnay, Pinot Gris and Columna were chosen, and among the red ones, the varieties were chosen: Cabernet Sauvignon, Fetească Neagră and Pinot Noir. White, pink or red, served as the basis for macerations and therapeutic vegetable decoctions. Medicinal wines are said to be elixirs with beneficial effects on health only if they are prepared correctly. Experts say that the vegetal parts used should be crushed, then used a natural wine, of high quality, free of impurities. From washing to drying, then mixing with special wooden spoons to storage in dark bottles, hermetically sealed and conditioned in areas with a constant temperature, necessarily requiring an alcohol of at least $40 \%$ alcohol, all must be observed. Once obtained, like the wine with medical application, this acquires well-defined active principles and must be consumed slowly, in small sips.

\section{The determination of sugars}

The analyzes are successive, every 3 days or 5 days, from the entry of the grape in the leek. The determination of sugars can be done with the help of the refractometer, which is based on the physical principle of refraction of light rays through a prism, on which is placed a film of liquid, must, with different density, according to the sugar content [3]. 


\section{Determination of total acidity}

The titratable acidity or total acidity is the totality of the acidic substances present in the wine and can be titrated with an alkaline solution in the presence of an indicator which turns to $\mathrm{pH}$ 7. The acidic substances found in the wine are organic acids or acidic salts.

The dosage of the titratable acidity is done titrimetric Take $10 \mathrm{~mL}$ of the analyzed white wine with a pipette and transfer to an Erlenmeyer flask. The new wine is heated to 40 degrees before titration in the sea bath to remove carbon dioxide and sulfur dioxide, which increase the titratable acidity. Then cool and add $10 \mathrm{~mL}$ of distilled water and 1 drop of $1 \%$ phenolphthalein. Titration takes place: from a burette add solution $\mathrm{NaOH} 0.1 \mathrm{~N}$ drop by drop, stir the contents until pale pink is formed. It should persist for 1-2 minutes. Phenol red or red litmus paper with a turning $\mathrm{pH}$ can also be used as an indicator solution.

To red wines, over $10 \mathrm{~mL}$ of wine add a double amount of distilled water or even more, to open the red. It is then titrated to neutralization, ie until olive or muddy green appears. The calculation of acidity is expressed in grams per liter in sulfuric acid or tartaric acid. There is also a limit to the acidification of wines. The maximum limit is $2.50 \mathrm{~g} / \mathrm{L}$ expressed in tartaric acid. [4].

N X F X0.0049 X 100 = acidity g / l expressed as sulfuric acid

To express the acidity in sulfuric acid, multiply the number of milliliters of alkaline solution used to neutralize the wine $(\mathrm{N})$ by the solution factor $(\mathrm{F})$, then by the equivalent in sulfuric acid -0.0049 and by 100 .

\section{Determination of alcohol from wine}

The main component of wine is ethyl alcohol from a legislative point of view and is the result of alcoholic fermentation of must sugars. Ethyl alcohol is a saturated, monohydric aliphatic alcohol, a colorless, flammable liquid with a specific taste and odor, with a boiling point of 78.4 density 0.7894 which has the formula: $\mathrm{C}_{2} \mathrm{H}_{5} \mathrm{OH}$

The alcoholic strength of a wine can be determined by the ebulioscopic method. Other methods may be used, provided that the wine has been previously distilled. In this case, the alcohol is determined on the distiller obtained, with the help of the alcohol meter, the pycnometer, of the Mohr - Westphall balance.

The dosing of alcohol with the ebulioscope is based on the fact that water and wine have different boiling temperatures. The mixture boils between $78.4^{\circ} \mathrm{C}$ and $100^{\circ} \mathrm{C}$ at normal pressure. Water boils at 100 degrees Celsius, while wine at 92.4 degrees Celsius. Wine, like water, is evacuated by boiling, and the division inscribed on the mobile disk remains on the ebuliometer disk. By mathematical calculations it is established at the respective temperature which alcoholic degrees correspond on the metal plate. The dosing of alcohol from wine by distillation consists in extracting the 
alcohol by distillation and determining the amount of alcohol using an alcohol meter or pycnometer.

\section{Determination of polyphenols content}

The dosage of polyphenols can be performed by the Folin-Ciocâlteau method, using the European Pharmacopoeia, 10th Edition. The results obtained from reading the absorbances of the samples on the spectrophotometer can be taken from the calibration curve. This is done by reading the absorbances of the increasing concentrations of gallic acid. The determination is made with the VWR UV-6300PC Spectrofotometru. The polyphenols can be oxidized by the Folin-Ciocâlteau reagent to form a blue oil with a maximum absorption at $750 \mathrm{~nm}$.

Determination of anthocyanins content

Total anthocyanins were determined by measuring the A520 of the aqueous phase using a VWR UV-6300PC spectrophotometer. All wine samples were made in triplicate.

\section{Results and Discussion}

\section{Grapes}

And if other foods contain sugars in the form of disaccharides or polysaccharides, requiring an expenditure of energy by the body, grapes provide sugars in the form of directly absorbable and usable. For this reason, grapes are suitable for those who consume a large amount of energy in a short time. In Figure 1 was presented the sugars (glucose and levulose) in grapes. The determination can also be done with the help of mustimeters, which are in fact aerometers or densimeters for liquids heavier than distilled water.

Grapes contain reducing sugars, malic acid, succinic acid, tanoids, flavonic derivatives, lecithin, choline, leucine, wax and minerals. When fresh, grapes contain 72-73\% water, sugars 16-22\% (glucose and levulose), free tartaric acid, other organic acids $0.30-0.35 \%$, mineral salts (potassium, manganese, zinc, bromine, calcium, sodium, iron and magnesium oxide, chlorine, silicon, phosphoric acid, iodine, arsenic), provitamin B (0.06 mg per 100 g), vitamins B1, B2, B5, C /7 mg /100 g, vitamin E and $P$ factors (vascular protectors), enocinamine (black grape dye with tonic effect), tannins in the skin (1.2\%), etc. They can be an alternative to nutrition. The beneficial effects of grapes can be highlighted. Grapes have a tonic energy appetizer, remineralizing, vitaminizing, diuretic, but also antitoxic, stimulant and laxative liver decongestant (1). Grapes have a cholagogue effect (favors the elimination of bile), eliminator of uric acid, with properties in regulating metabolic functions, hypotensive and lowering blood cholesterol. Grapes are used in anemia, pregnancy, obesity, in demineralization, rheumatism, intoxication, hepatitis, atherosclerosis, hemorrhage.

According to T. Martin, grapes have an energetic action. "The sugars (glucose and levulose) in grapes and must represent a strictly necessary element for muscle effort 
and become indispensable for the function of the heart muscle and the central nervous system" [5]

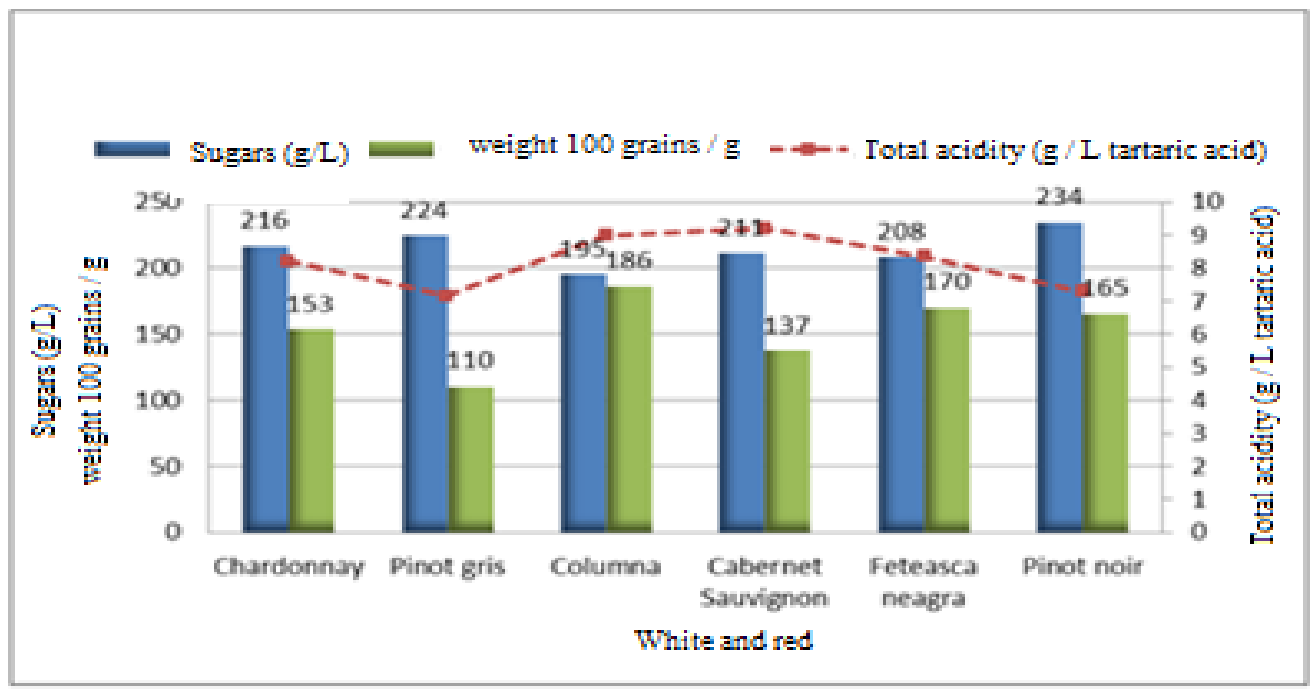

Figure 1 Grape quality, 2019 harvest

Grapes also have an alkalizing role, explained by the fact that they have a high content of acids that determine the formation of carbonates and phosphates. Therefore, grapes maintain the acid-base balance.

The remineralizing role of grapes is given by the varied content of mineral salts and ions. Grapes are a good diuretic and, being poor in albumin and sodium chloride, are indicated in kidney disease.

Rich in cellulose, grapes are also indicated in intestinal diseases, having a laxative action. "Some components of the must act in the same sense: tartrates, due to the fact that they are practically unabsorbed by the intestinal mucosa, sulfates and magnesium" [5]. Grapes are also indicated in putrefaction colitis, due to the intensification of fermentation processes.

Both grapes and must produce a stimulation of liver function, with carbohydrates having a protective role on the cells, while phosphates and potassium influence the formation of liver glycogen.

And some dermatitis can be treated with grapes, as can acne and hives. In addition, grapes are part of the diet of diabetics, due to the presence of fructose, whose metabolism is independent of insulin.

\section{The wines}

The wines from the Dobrogea hills, showed us the physico-chemical analyses, have extraordinary properties and can be used for their superior healing qualities. 
The dynemic of sugar was presented in Figure 2 - (Fig. 2.a for white wine and Fig. 2.b. for red wine). The dosing of sugars is an extremely important stage, because it is taken into account to determine the time of ripening of the grapes.

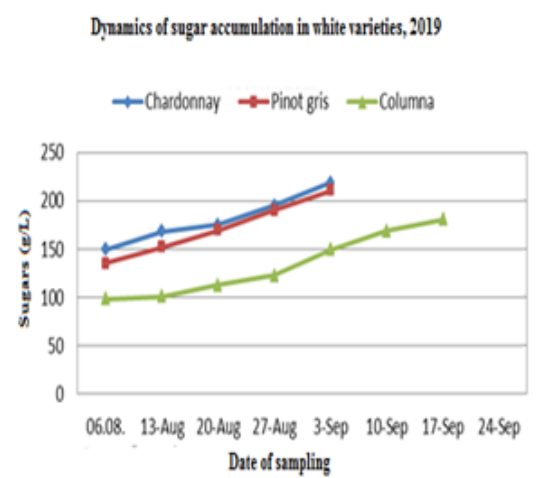

White wines

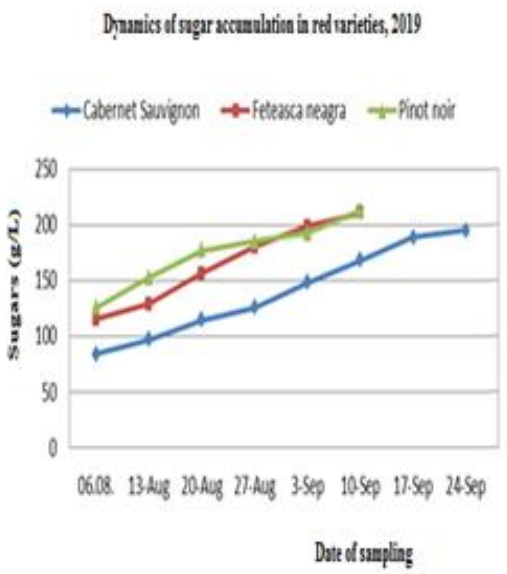

Red wines

Figure 2 Evolution of sugar accumulation in white and red wines

Sweetening is a common process in the wine industry. This can increase the sugar content and is made with grape must. It can also be made with concentrated grape must or rectified concentrated grape must. Determining sugars is perhaps one of the most important parameters, along with acidity. The amount of sugars helps us not only to choose the optimal time of harvest, but also to establish the vinification strategy.

The dynamic of total acidity in wine was presented in Figure 3. The wine contains tartaric acid and malic acid. In wine, some of the acids come from the must, and another part is formed in the process of alcoholic fermentation or other fermentations, but also during storage. Among the acids that come from the must we find:

Tartaric acid (0.4 - $5.6 \mathrm{~g} / \mathrm{L}$ ) and constitutes $2 / 3$ of the total acids found in wine. Tartaric acid is what imparts to the wine the specific taste of wine, in the presence of alcohol, the solubility decreasing

Malic acid is found in wines that have been made from grapes that have not fully ripened

Citric acid is found naturally in wine. It is added only if the wine has low acidity for improvement [3] 


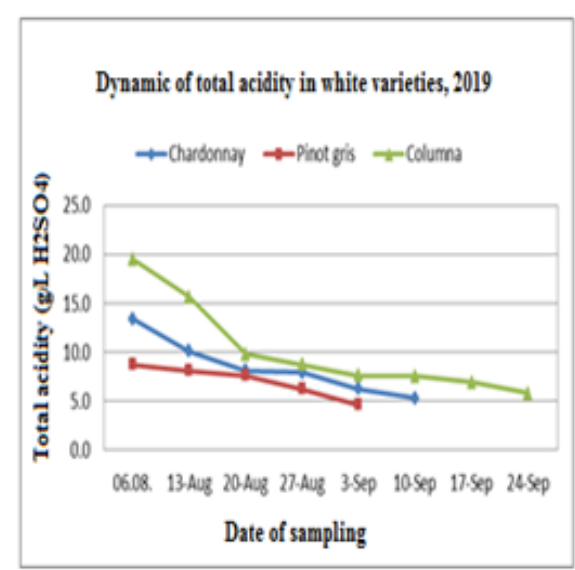

White wines

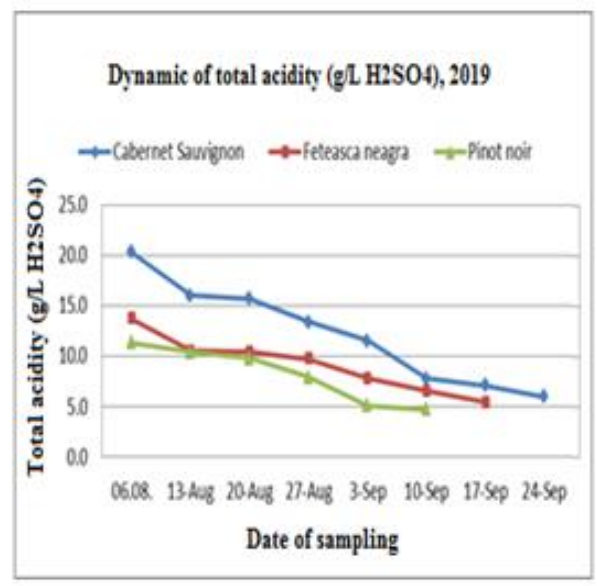

Red wines

Figure 3. Evolution of total acidity in white and red varieties in 2019

Acids are also formed during normal alcoholic fermentation:

Succinic acid $0.25-1.5 \mathrm{~g} / \mathrm{L}$

Lactic acid $0.5-6 \mathrm{~g} / \mathrm{L}$

Acetic acid, which is a normal product of the fermentative activity of yeasts in alcoholic fermentation [4].

Normal acetic acid values should be:

0.2 - $0.6 \mathrm{~g} / \mathrm{L}$ for white wines

$0.6-0.9 \mathrm{~g} / \mathrm{L}$ for red wines.

The determination of the acidity of the must and the wine is made in order to decide which is the date of harvest for the type of wine proposed to be obtained. Determining the acidity helps us to correct the acidity of the musts, but also that of the more acidrich wines. Determining the acidity also helps us to correct wines with a high acidity, in order to ensure the health of the wine. We know that acids, along with alcohol and tannin decide to preserve and age the wine, to determine the quality of the wine. [3]. The total acidity of the wine varies between 3 and $6 \mathrm{~g} / \mathrm{L}$ sulfuric acid.

It should be noted that the determination of total acidity is made only for wines in which we have absolutely no carbon dioxide. If present, it is removed with a vacuumresistant vessel. Volatile acidity is also taken into account. Volatile acidity refers to all acids that evaporate at 100 degrees Celsius. In normal wines, volatile acidity is formed as a by-product in the fermentation process and is given by acetic acid. 
A normal wine contains $0.3-0.6 \mathrm{~g} / \mathrm{L}$ volatile acidity, expressed as sulfuric acid. If the volatile acidity exceeds the ranges of $0.5-0.8 \mathrm{~g} / \mathrm{L}$ for white wines and $1.5-2 \mathrm{~g} / \mathrm{L}$ for red wines, then the wines acquire a sour, unpleasant taste, which irritates the taste buds, making them uneatable [3]. The law prohibits the sale of white wines that exceed $1 \mathrm{~g} / \mathrm{L}$ or red wines that exceed $1.25 \mathrm{~g} / \mathrm{L}$. The volatile acidity of the wine is what tells us how healthy a wine is. The determination of volatile acidity is therefore necessary to protect the wine from vinegar and to control its bouquet.

The alcoholic strehgth of wine was presented in Figure 4. After water, ethyl alcohol is the main liquid element of wine, forming in the process of alcoholic fermentation of must. Under the action of yeast, natural sugar decomposes into approximately equal amounts of alcohol and carbon dioxide. Wines can reach a concentration in alcohol between 8-14 degrees, sometimes even 15 degrees.

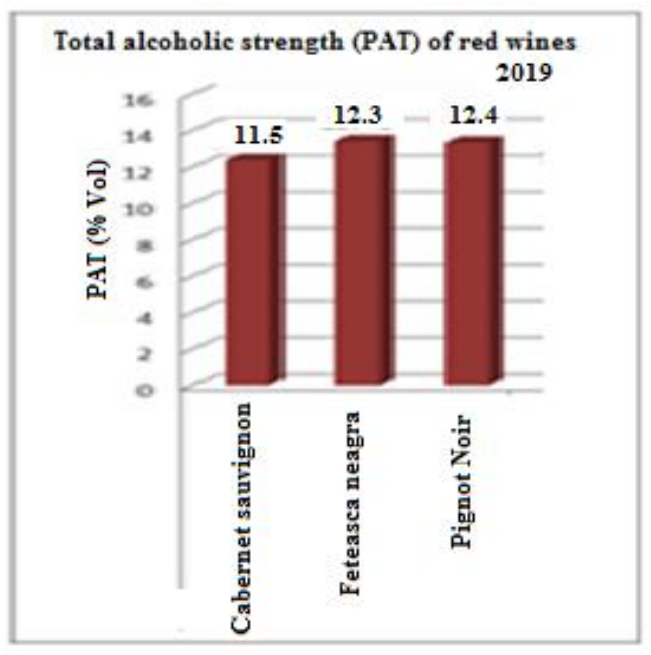

Red wines

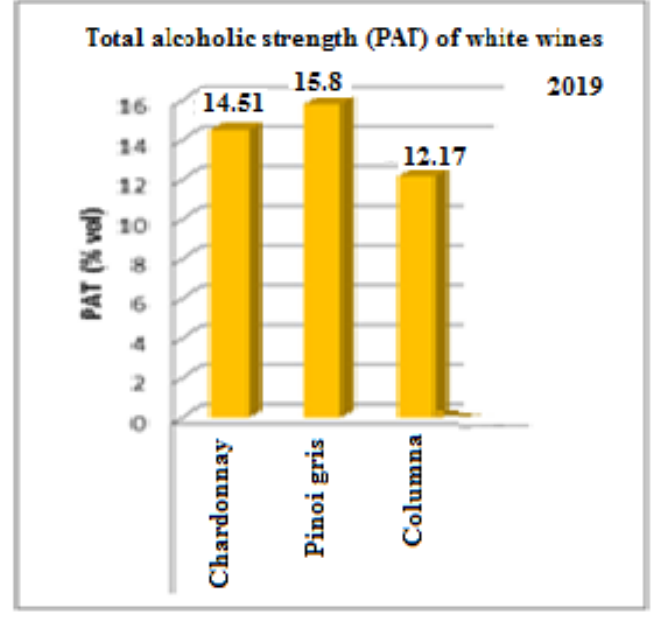

White wines

Figure 4 Alcohol concentration ( $\%$ vol) for red and white wines

The alcoholic strength resulting from a normal fermentation may not exceed 15 degrees [6].

The alcoholic strength of the wine or the alcoholic strength is the amount of one hundred percent pure ethyl alcohol in ml contained in $100 \mathrm{~mL}$ of wine. The alcohol content of a wine can vary during its life. As they age, wines usually lose alcohol by evaporation. The higher the storage temperature, the lower the alcohol content of the wine by evaporating the alcohol [3]. The alcohol content of a wine variety is equally influenced by the soil (limestone gives wines richer in alcohol than sands), the type of vine, the climate, the year of harvest (the warm years give a wine with a higher degree high alcohol). 
Wine alcohol is a good preservative, alcohol content being considered a quality criterion. The words "wine", "spirit", "which goes up to the head" characterize wines with a high alcohol content [6].

Polyphenols are compounds that are a fine part of the group of phenols, which are found in wines, in small quantities in white wines, large in red wines, having antioxidant properties. A phenol group is a compound with a hydroxyl group $(-\mathrm{OH})$ attached to an aromatic carbon group. Depending on the number and arrangement of these phenolic groups, different polyphenolic compounds will have different biological and chemical effects in the body. In Figure 6 was presented total phenols content in red wines.

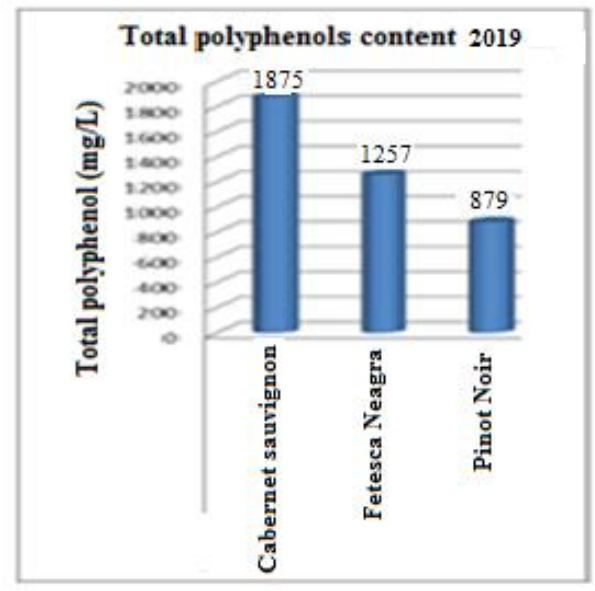

Figure 6 Total polyphenols in red wines

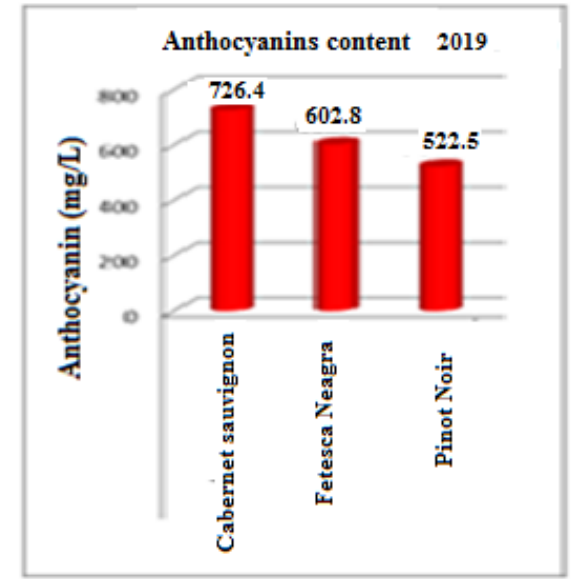

Figure 7 Anthocyanins content in red wines

Polyphenols reduce oxidative stress, have anti-inflammatory, immunoprotective and anti-cancer properties. One of the best known polyphenols is resveratrol, which is found in red grapes. Hence the recommendation of the French to consume a glass of red wine daily.

Polyphenols are often used by the ester industry, providing elasticity to the skin and preventing the unwanted appearance of wrinkles. In addition, polyphenols are used because they give ferments to the skin, but also help regenerate cells and tissues. Moreover, they have the ability to stimulate the synthesis of collagen and elastin in the skin. Polyphenols can be consumed in the form of antioxidant supplements, and are generally obtained from grape seed extract. The polyphenolic profile of a variety represents the genetic potential.

Anthocyanins content in red wines

In Figure 7 was presented Anthocyanins content in red wines. The highest anthocyanin content was recorded, the Cabernet Sauvignon variety, and the lowest in 
Pinot Noir in 2019. Anthocyanins have a strong antioxidant role, they prevent the destructive action of free radicals on protein cells and DNA. Protects the body against serious diseases such as cancer or heart disease.

\section{Therapeutic indications}

A glass of white wine covers $10 \%$ of the body's magnesium and $20 \%$ of the body's manganese. It contains 120 more substances, the most important being vitamins and minerals. It is recommended to be consumed in the evening, in small quantities. To maintain our silhouette, mineral water spray (which halves calories) is recommended. Red wine belts prevent allergies. Red wine should be drunk daily, a glass of $125 \mathrm{~mL}$. The first signs of healing appear after 3-4 weeks of treatment, and as a time of year, the spring and autumn months are ideal. At the same time, red wine is also recommended for people with allergic asthma. The medical explanation for the success of this procedure is that red wine stimulates the formation of histamines, which are nothing more than the antibody produced by the body to defend against allergies.

In patients with atherosclerosis, polyphenols synergistically inhibit oxidative stress in subjects receiving red and white wine [7].

In the case of kidney disorders, it has been established that there is a link between wine and disease [8]. The analyses performed on the subjects showed an association of moderate wine consumption with a low risk of cardiovascular and neurological diseases. The health benefits of moderate wine consumption can be partly attributed to its antioxidant properties. The antioxidant defense system of the kidney is increased after chronic exposure to moderate amounts of wine. This view is partially supported by direct evidence that wine and antioxidants isolated from red wine, as well as other antioxidants, significantly attenuate or prevent oxidative damage to the kidney [8].

British researchers have analyzed the effects of resveratrol on the human body and concluded that this powerful antioxidant in red wine can reduce the incidence of colon cancer by up to $50 \%$, but also intestinal tumors [2]. Harvard specialists are convinced that the elixir of youth is found in wine. They were the first to notice the anti-aging effect of red wine [2].

Chinese experts say that diluted ethanol in wine enhances cellular phenotypic changes in human breast cancer and esophageal carcinoma, but also that cell growth suppression, colony formation and Pol III genes by mature wines are much stronger than young wines [9].

\section{Conclusion}

We started from the idea that there should be common elements between the health field and the pharmaceutical industry, on the one hand, and Murfatlar wine, on the other hand. 
Following the laboratory analyses, we discovered not only that all the chosen wines correspond to the quality criteria in the field, but also have superior properties. Chardonay, Pinot Gris or Columna, among the white wines, or Cabernet Sauvignon, Fetească Neagră or Pinot Noir, whichever you would like to choose, you will have an ally on your side.

Whether we are talking about wine polyphenols that protect blood vessels, or we use it as a remedy for cellular or physical balance or it serves as a basis for macerations and herbal therapeutic decoctions, we say that Bachus liqueur is a true elixir with beneficial effects on the our body.

Funding: This work was supported by the Romanian Ministry of Research an Innovation, UEFISCDI, project No. PN-III-P1-1.2-PCCDI-2017-0701, grant no. 85PCCDI/2018. APC was funded by the Romanian Ministry of Research an Innovation, Institutional Performance Projects for Excellence Financing in RDI, grant No. 22PFE/2018

\section{References}

[1] Arnold von Eckardstein, Atherosclerosis, september 2016, volume 188, "Polyphenols synergistically inhibit oxidative stress in subjects given red and white wine".

[2] Chen Solin, Yunfeng Yi, "The influences of red wine in phenotypes of human cancer cells", Vol. 702, 20 June 2019, p. 194-204

[3] Geoergescu M., Pomohaci N., Viticulture and vinification, Didactic and Pedagogical Publishing House, Bucharest, 1971. *** Methodological norms for the application of the Law on Vine and Wine in the system of common organization of the wine market no. 244/2002, published in the Official Monitor Romania, Part I, no. 595 of August 23, 2010

[4] Martin T., Turkish I, Georgescu M, Martin, D, Grape and must treatment, AgroForestry Publishing House, Bucharest.

[5] Petrescu E., Terapia cu struguri şi vinuri medicinale, Editura Polirom, Iaşi, p.28, 2002. ${ }^{* * *}$ Study backs alcohol UK limit of six glasses of wine a week, 13 aprilie 2018, www.bmj.com

[6] Rodrigo R., , Gonzalo R., Renal damage mediated by oxidative stress: a hypothesis of protective effects of red wine, în Radical Biology and Medicine, Volume 33, Issue 3, 1 August 2002.

[7] Toma V., Dictionary of the winemaker, Alma Publishing House, Craiova, 2001. 